\title{
Caffeine exposure affects barpressing
}

\author{
JENNIFER O'LOUGHLIN, J. CHRIS GRAVES, and STEPHEN F. DAVIS \\ Emporia State University, Emporia, Kansas \\ and \\ RANDOLPH A. SMITH \\ Ouachita Baptist University, Arkadelphia, Arkansas
}

\begin{abstract}
Three breeding pairs of rats were randomly assigned to one of three caffeine-exposure conditions (pre- and postnatal, postnatal, or control) to assess the effects of caffeine on barpressing. Pre- and postnatally exposed pups received $.50 \mathrm{mg} / \mathrm{ml}$ of caffeine in their drinking water throughout gestation and thereafter. Postnatal pups received caffeine-adulterated drinking water only following birth. Control pups received plain tap water both pre- and postnatally. At 45 days of age, a series of 15 daily, 8-min barpress sessions began. The results indicated that postnatal-only exposure hindered performance, whereas pre- and postnatal exposure facilitated performance.
\end{abstract}

Previous research has suggested that caffeine exposure may initially facilitate performance. This facilitation is typically followed by a performance decrement. Such increments, followed by decrements, are observed on both a between- and a within-session basis. For example, Loke and Meliska (1984) reported that ambulation and rearing behavior were reduced during the 2 nd hour of a testing session. Mueller, Saboda, Palmour, and Nyhan (1982) reported that caffeine exposure enhanced the frequency of tight circling behavior during early training sessions but depressed this behavior during later sessions. Similarly, Holloway and Thor (1982) reported increases, followed by decreases, in the locomotor behavior of caffeineexposed rats.

More recently, Cathey, Smith, and Davis (1993) reported that caffeine exposure begun during gestation and continued throughout training resulted in facilitation of maze performance during the early stages of training but either had no effect or hindered performance during the later stages. These investigators also found that caffeine exposure resulted in a general reduction in barpress behavior. Because caffeine was administered both prenatally and postnatally in the Cathey et al. (1993) study, "The question of whether caffeine-related decrements occurred because of prenatal or postnatal caffeine exposure" remained "unanswered" (p. 50). The present study addressed this question.

\section{METHOD}

\section{Subjects}

Three adult male and 3 adult female rats were randomly selected from the colony maintained at Emporia State University to form three breeding pairs. In turn, the breeding pairs were randomly assigned to one

Reprint requests should be directed to R. A. Smith, Department of Psychology, Ouachita Baptist University, Arkadelphia, AR 71998-0001 or S. F. Davis, Department of Psychology, Emporia State University, Emporia, KS 66801-5087. of the three following conditions: pre- and postnatal, postnatal, and control. The breeding pairs were housed together for 10 days and then separated. The pups were weaned at 21 days of age and placed in individual cages. Eight pups participated under the control (CON) conditions, 10 pups served in the prenatal and postnatal (PREP) group, and 14 pups served in the postnatal (POST) group.

\section{Apparatus}

Barpress training took place in a Lafayette operant conditioning chamber (Model 81335). Reinforcement consisted of 45-mg Noyes pellets.

\section{Procedure}

Upon formation of the breeding pairs, the PREP animals received exposure to a solution of $.50 \mathrm{mg} / \mathrm{ml}$ caffeine in plain tap water, whereas the POST and CON pairs received plain tap water. These conditions remained in effect until the birth of the pups. At that time, both the PREP and POST dams were allowed to consume the caffeine solution on an ad-lib basis. The CON dam and pups consumed plain tap water throughout the experiment.

A 15-day barpress training phase began when the pups were 45 days old. Twenty-four hours prior to the start of barpress training, all food was removed from each subject's cage. During this phase, each subject received an 8-min session in the operant chamber. The order for running subjects was randomized daily. Barpresses were reinforced on a fixed ratio schedule, with reinforcement after each response. One hour following the completion of each daily session, the animals were allowed free access to Purina Laboratory Chow for $15 \mathrm{~min}$.

\section{RESULTS AND DISCUSSION}

Group mean barpresses for the 15 daily sessions are shown in Figure 1. Analysis of variance of these data yielded significance for days $[F(14,406)=89.76, p<$ $.001]$ and groups $\times$ days $[F(28,406)=2.13, p=.001]$. Newman-Keuls tests, employed to probe the significant interaction, indicated that Groups PREP and CON made significantly more responses $(p<.05)$ than did Group POST on Days 8-12 and 14. In addition, Group PREP made significantly more responses $(p<.05)$ than did Group CON on Days 9-12. Group CON made significantly more responses $(p<.05)$ than did Groups PREP and POST on Day 15. 


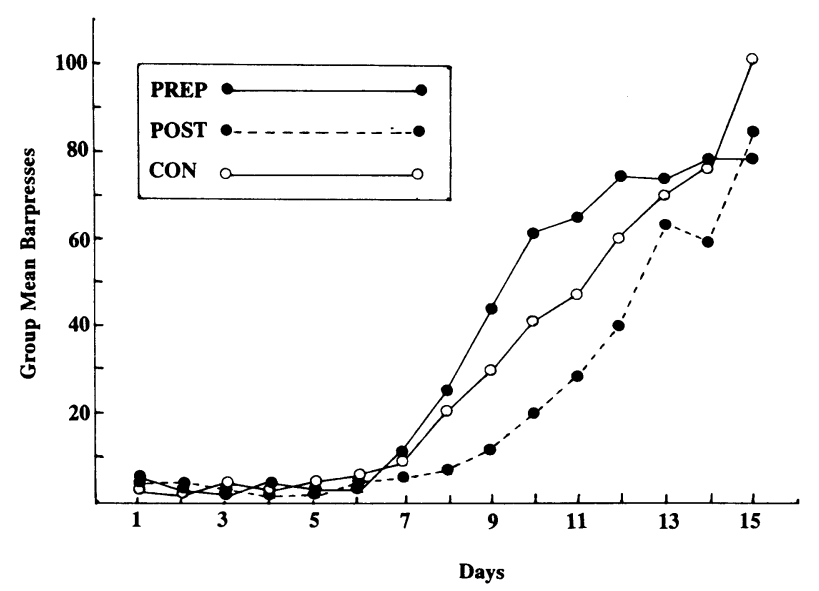

Figure 1. Group mean barpresses for animals receiving prenatal caffeine exposure (Group PREP), postnatal caffeine exposure (Group POST), and exposure to plain tap water (Group CON).

Clearly, caffeine exposure had an effect on barpressing behavior. If one considers just the performance of Groups
POST and CON, a conclusion similar to that of Cathey et al. (1993) is prompted; caffeine exposure resulted in reduced levels of barpressing. However, when all three groups are considered, a different picture emerges. Preand postnatal exposure to caffeine (Group PREP) resulted in performance enhancement, whereas postnatal exposure (Group POST) served to depress performance.

\section{REFERENCES}

Cathey, N. R., Smith, R. A., \& Davis, S. F. (1993). Effects of caffeine on rats' barpress and maze performance. Bulletin of the Psychonomic Society, 31, 49-52.

Holloway, W. R., \& Thor, D. (1982). Caffeine sensitivity in the neonatal rat. Neurobehavioral Toxicology \& Teratology, 4, 331-333.

LOKE, W. H., \& MELISKA, C. J. (1984). Effects of caffeine and nicotine on open-field exploration. Psychological Reports, 55, 447-451.

Mueller, K., Saboda, S., Palmour, R., \& Nyhan, W. L. (1982) Self-injurious behavior produced in rats by daily caffeine and continuous amphetamine. Pharmacology, Biochemistry \& Behavior, 17, 613-617.

(Manuscript received April 17, 1993.) 